\title{
Sexualidade e a imagem corporal em idosas: revisão integrativa
}

\section{Sexuality and a body image in elderly: integrative review}

\author{
Juliana Cordeiro Carvalho' $\bullet$ Monique de Freitas Gonçalves Lima ${ }^{2} \bullet$ Rogério Dubosselard Zimmermann ${ }^{3} \bullet$ Már- \\ cia Carrera Campos Leal ${ }^{4}$ Maria da Conceição Lafayette de Almeida ${ }^{5}$ Nicole Vasconcelos de Souza $^{6}$
}

\section{RESUMO}

Objetivo: analisar a relação entre a imagem corporal e a sexualidade em idosas. Método: foi realizada uma revisão integrativa dos artigos publicados em inglês, espanhol e português entre 2013 a 2018, nas bases de dados: LILACS, PUBMED, BDENF - Enfermagem, IBECS, Index Psicologia - Periódicos técnico-científicos, SCOPUS e Web of Science, realizando o cruzamento dos descritores Aged, Sexuality and Body Image. Resultados: Após aplicação dos critérios de inclusão e exclusão resultaram dois artigos de pesquisa qualitativa em que se evidenciou ser a auto-imagem um fator que pode ou não influenciar na sexualidade nas idosas. Conclusão: Ficou evidente a insuficiência de estudos relacionados ao tema.

Palavras-chave: Idoso. Imagem corporal. Sexualidade.

\section{SUMMARY}

Objective: to analyze the relationship between body image and sexuality in the elderly. Methods: Na integrative review of articles published in English, Spanish and Portugues between 2013 and 2018 was carried out in the datebases: LILACS, PUBMED, BDENF - Nursing, IBECS, Index Psychology - Scientific and technical journals, SCOPUS and Web of Science, used as descriptors: aged, sexuality and body image. Results:After applying the inclusion and exclusion criteria, two articles of qualitative research resulted in self-image being a fator that influences sexuality in the elderly. Conclusion:The lack of studies related to the topic was evident.

Keywords: Elderly. Body Image. Sexuality.

I Mestra pelo Programa de Pós-Graduação em Gerontologia (PPGERO) na Universidade Federal de Pernambuco (UFPE). Graduação em Fisioterapia. E-mail: julianacordeirocarvalho@hotmail.com - Currículo Lattes: http://lattes.cnpq.br/875I 225 I97550845

2 Mestra pelo Programa de Pós-Graduação em Gerontologia (PPGERO) na Universidade Federal de Pernambuco (UFPE). Graduação em Enfermeira. E-mail: monique_freitas@hotmail.com - Currículo Lattes: http://lattes.cnpq.br/6332269/24307992

3 Doutor e Professor pelo Programa de Pós-Graduação em Gerontologia (PPGERO) na Universidade Federal de Pernambuco (UFPE). Graduação em Odontologia. E-mail:rdzlegal@gmail.com - Currículo Lattes: http://lattes.cnpq.br/81527837932921 I8

4 Doutora e Professora pelo Programa de Pós-Graduação em Gerontologia (PPGERO) na Universidade Federal de Pernambuco (UFPE), Graduação em Odontologia. E-mail: marciacarrera@hotmail.com - Currículo Lattes: http://lattes.cnpq.br/9565468344357009

5 Doutora e Professora pelo Programa de Pós-Graduação em Gerontologia (PPGERO) na Universidade Federal de Pernambuco (UFPE), Graduação em Sociologia. E-mail: celafayette@hotmail.com - Currículo Lattes: http://lattes.cnpq.br/9320385747508007

6 Pós-Graduada em Gerontologia pelo Instituto de Desenvolvimento Educacional (IDE). Graduação em Serviço Social - E-mail: nicole_souza05@hotmail.com 


\section{INTRODUÇÃO}

O envelhecimento é caracterizado pela deterioração dos sentidos e mudanças visíveis, relacionadas à idade e aparência física que inclui rugas, cabelos grisalhos, manchas na pele, diminuição da massa muscular, entre outros. Esses sinais podem causar sentimentos de vergonha, alienação, raiva e inferioridade, dificultando a aceitação da imagem corporal ${ }^{(1)}$.

Assim, é importante abordarmos a imagem corporal, como um fenômeno que envolve aspectos cognitivos, afetivos, sociais/culturais e motores. Está associado com o conceito de si próprio e é influenciável pelas interações entre o ser e o meio em que vive. $O$ seu processo de desenvolvimento está associado às concepções sociais e culturais. $O$ ideal de estética corporal foi sofrendo alterações à medida que as normas e os comportamentos foram mudando(2).

No caso da mulher idosa, as transformações em seu corpo e as forças opressoras culturais sustentam o argumento da impossibilidade de serem vistas como atraentes e, como consequência disto, questionam sua capacidade de sedução e de vivência plena da sexualidade ${ }^{(3,4)}$. $O$ culto ao corpo jovem, idealizado pela mídia, perpetua a convicção de que a sexualidade esteja ligada à beleza jovial ${ }^{(5)}$.

Portanto, o corpo que envelhece, e para a maioria dos comportamentos sociais, geralmente não produz mais interesse e é retratado sem desejo, sem atração física e em um estado de declínio.Assim, as pessoas que envelhecem são, na maioria das vezes, rotuladas como assexuadas ou incapazes de sentirem desejo, tornando evidente que o assunto da sexualidade do idoso está impregnado de rótulos, tabus e preconceitos ${ }^{(6)}$, expressando os fatores socioculturais e históricos envolvidos.

O problema do estudo se estabelece a partir do momento em que a imagem corporal do idoso possa refletir de forma negativa no comportamento sexual. $\mathrm{Na}$ tentativa de contribuir para a ampliação do conhecimento das ações na saúde, este estudo objetivou analisar na literatura a relação entre a imagem corporal e a sexualidade em idosas.

\section{MÉTODO}

Trata-se de uma revisão integrativa, considerada um método de estudo que possibilita a síntese do conhecimento de um determinado assunto, além de apontar lacunas, que precisam ser preenchidas com a realização de novos estudos ${ }^{(18)}$. Neste artigo, procuramos contextualizar o conhecimento da relação entre a sexualidade e a imagem corporal em idosas.

Para o desenvolvimento da presente revisão foram percorridas as seguintes etapas: estabelecimento da questão norteadora; definição dos critérios de inclusão e exclusão de estudos; busca dos artigos e seleção da amostra; ava- liação dos estudos incluídos; interpretação dos resultados; discussão e apresentação da revisão integrativa ${ }^{(7)}$.

Para orientar a pesquisa, elaborou-se a seguinte pergunta: Quais as evidências científicas publicadas entre os anos de 2013 à 2018 que retratam a relação da imagem corporal com a sexualidade em idosas?

A coleta de dados ocorreu entre os meses de setembro a novembro de 2018 e foram utilizadas na seleção dos artigos, as seguintes bases de dados eletrônicos: Literatura Latino-Americana em Ciências da Saúde (LILACS), US National Library of Medicine National Institutes of Health (PUBMED), Base de Dados em Enfermagem (BDENF - Enfermagem), Indice Bibliográfico Español en Ciencias de la Salud (IBECS), Index Psicologia - Periódicos técnico-científicos, Scopus e Web of Science. Os artigos foram selecionados nas seguintes línguas: inglês, espanhol e português. Empregaram-se os Descritores em Ciências da Saúde (DeCS):Aged, Body Image, Sexuality.

Os critérios de inclusão estabelecidos para a seleção dos artigos foram: estudos que abordam idosas, artigo original; responder a questão norteadora; ter disponibilidade eletrônica na forma de texto completo, ter sido publicado no período supracitado nos idiomas português, inglês e espanhol. Foram excluídos: pesquisas que consideraram indivíduos com idade inferior a 60 anos e estudos repetidos em uma ou mais bases de dados.

Para avaliar a qualidade dos estudos selecionados foram utilizados dois instrumentos: o primeiro, adaptado do Critical Appraisal Skills Programme (CASP) - Programa de Habilidades em Leitura Crítica, integrante do "Public Health Resource Unit" (PHRU). O instrumento é composto por 10 itens (máximo 10 pontos), abrangendo: I) objetivo; 2) adequação metodológica; 3) apresentação dos procedimentos teóricos e metodológicos; 4) seleção da amostra; 5) procedimento para a coleta de dados; 6) relação entre o pesquisador e pesquisados; 7) consideração dos aspectos éticos; 8) procedimento para a análise dos dados; 9) apresentação dos resultados; 10) importância da pesquisa. Os estudos foram classificados de acordo com as seguintes pontuações: 06 a 10 pontos (boa qualidade metodológica e viés reduzido), e mínima de 5 pontos (qualidade metodológica satisfatória, porém com risco de viés aumentado) ${ }^{(8)}$.

O segundo instrumento utilizado correspondeu à Classificação Hierárquica das Evidências para Avaliação dos Estudos' , e contempla os seguintes níveis: I) revisão sistemática ou metánalise; 2) ensaios clínicos randomizados; 3) ensaio clínico sem randomização; 4) estudos de coorte e de caso-controle; 5) revisão sistemática de estudos descritivos e qualitativos; 6) único estudo descritivo ou qualitativo; 7) opinião de autoridades e/ou relatório de comitês de especialidades. Ao final da análise destes dois instrumentos, totalizaram-se dois artigos. 


\section{RESULTADOS}

Na Tabela I, verificam-se os resultados encontrados a partir da busca pelos descritores, conforme cada base de dados.

Os idiomas encontrados nos artigos foram inglês e português. As revistas foram a International Psychogeriatrics e Saúde Soc. Quanto ao tipo de delineamento de pesquisa, evidenciou-se na amostra dois estudos qualitativos. Dessa forma, em relação à força das evidências obtidas nos artigos, os dois encontraram-se com nível de evidencia Vl.

Os artigos foram caracterizados a partir de variáveis distribuídas em publico alvo cujos dados estão resumidos no quadro 01 .

TABELA 1 - Relação dos artigos selecionados conforme Base de dados. 2018.

\begin{tabular}{c|c|c|c|c}
\hline Bases de Dados & Encontrados & Pré-Selecionados & Excluídos & Selecionados \\
\hline Pubmed & 53 & 6 & 6 & 0 \\
\hline Lilacs & 5 & 1 & 0 & 1 \\
\hline Bdenf & 5 & 1 & 1 & 0 \\
\hline Ibesc & 5 & 1 & 0 & 0 \\
\hline Index Psicologia & 2 & 0 & 19 & 0 \\
\hline Scopus & 151 & 19 & 02 & 1 \\
\hline Web of Science & 112 & 03 & 29 & 2 \\
\hline Total & 333 & 31 & & 0 \\
\hline
\end{tabular}

Fonte: Pesquisa realizada em banco de dados online coletados no ano de 2018

QUADRO 01 - Distribuição das variáveis e as respectivas características da sexualidade e do exercício físico em idosos. 2018.

\begin{tabular}{|c|c|}
\hline Variáveis & Caracterização \\
\hline Publico Alvo & Mulheres ${ }^{1,2}$ \\
\hline Idades dos Idosos & Média de 66 anos $^{1} 60$ em diante ${ }^{2}$ \\
\hline Estado Marital & Relacionamentos longos e recém formados ${ }^{1}$ Viúvas solteiras ${ }^{2}$ \\
\hline Instrumentos de avaliação sobre a sexualidade & Entrevista Semiestruturada ${ }^{1},{ }^{2}$ \\
\hline Instrumento de avaliação da imagem corporal & Entrevista Semiestruturada ${ }^{1}{ }^{2}$ \\
\hline
\end{tabular}

QUADRO 02 - Apresentação da síntese dos artigos incluídos na revisão integrativa sobre sexualidade e imagem corporal em mulheres idosas. Recife, 2018.

\begin{tabular}{|c|c|c|c|}
\hline Título & Autor/ano/ País & Objetivo/tipo de estudo/metodologia & Resultado \\
\hline $\begin{array}{l}\text { 1. Old and desirable: } \\
\text { older women's accounts } \\
\text { of ageing bodies in } \\
\text { intimate relationships }\end{array}$ & $\begin{array}{l}\text { Rachel Thorpea, } \\
\text { Bianca Fileborna, } \\
\text { Gail Hawkesb, } \\
\text { Marian Pittsa and } \\
\text { Victor Minichiello, } \\
2015 \\
\text { Austrália }\end{array}$ & $\begin{array}{l}\text { Estudo qualitativo por meio de entrevistas } \\
\text { semi - estruturadas com } 20 \text { mulheres, } \\
\text { cujo objetivo foi estudar as subjetividades } \\
\text { sexuais através de relatos de mulheres } \\
\text { idosas australianas sobre seus corpos } \\
\text { e sobre experiências incorporadas no } \\
\text { contexto de relacionamentos íntimos de } \\
\text { longo prazo e recém-formados. }\end{array}$ & $\begin{array}{l}\text { O estudo revelou que, embora as } \\
\text { mulheres estivessem frequentemente } \\
\text { insatisfeitas com sua aparência, isso } \\
\text { era menos importante para elas nos } \\
\text { relacionamentos. Através dos relatos } \\
\text { das participantes indicou que apesar dos } \\
\text { corpos das mulheres mais velhas serem } \\
\text { enrugados e velhos, é possível ainda } \\
\text { serem desejáveis. }\end{array}$ \\
\hline $\begin{array}{l}\text { 2. A vivência da } \\
\text { sexualidade por } \\
\text { idosas viúvas e suas } \\
\text { percepções quanto à } \\
\text { opinião dos familiares a } \\
\text { respeito }\end{array}$ & $\begin{array}{l}\text { Souza, M.; Marcon, } \\
\text { Bueno, S.M.V.; } \\
\text { Carreira, L.; } \\
\text { Baldissera, V.D.A. } \\
2015 \\
\text { Brasil }\end{array}$ & $\begin{array}{l}\text { Tratou-se de uma pesquisa qualitativa, do } \\
\text { tipo exploratório- } \\
\text { Descritivo. Utilizou-se entrevista semi } \\
\text { - estruturada. As temáticas abordadas } \\
\text { foram: 1) A vivencia da sexualidade na } \\
\text { viuvez e sua submissão às normas e } \\
\text { regras sociais; 2) As idosas optam por } \\
\text { um novo companheiro; 3) A família apoia } \\
\text { o convívio social, mas não apoia novos } \\
\text { relacionamentos. } \\
\text { O objetivo foi descrever a vivência } \\
\text { da sexualidade por mulheres idosas } \\
\text { viúvas, frequentadoras de um Centro de } \\
\text { Convivência do Idoso. }\end{array}$ & $\begin{array}{l}\text { A sociedade impõe um pré-julgamento } \\
\text { nas mulheres idosas, levando-as a } \\
\text { modificarem a sua imagem corporal } \\
\text { para manter uma postura mais discreta. } \\
\text { Algumas relatam que por serem velhas já } \\
\text { não oferece "atrativos" Fora que também } \\
\text { tentam evitar falar e pensar em sexo, } \\
\text { por ser algo de "velha assanhada", além } \\
\text { de ser algo visto como atos feitos só por } \\
\text { jovens e adultos. }\end{array}$ \\
\hline
\end{tabular}




\section{DISCUSSÃO}

Nesta revisão, foram identificados dois tipos de abordagens sobre a relação entre a sexualidade e a auto estima em idosas. No primeiro artigo, concluiu-se que a aparência não é algo relevante e que por consequência não interfere na sexualidade das idosas estudadas. Já no segundo artigo revela que existe influencia da imagem corporal de idosas sobre a sua sexualidade, preferindo adotar uma postura discreta, além de evitarem falar sobre o sexo por poderem ser rotuladas como velhas assanhadas.

Tem-se considerado que a pessoa idosa seja desprovida de desejos e de vida sexual, como se os anos Ihe trouxessem uma inapetência neste aspecto vital do desenvolvimento humano. Entretanto, a literatura atual tem demonstrado não existirem razões fisiológicas que impeçam as pessoas idosas, em condições satisfatórias de saúde, de apresentarem uma vida sexual ativa ${ }^{(I I)}$.

A vivência da sexualidade é um modo de expressar carinho, afeto, admiração por alguém; é autoafirmação de si, de seu corpo, autoestima elevada. A imagem corporal está diretamente ligada com a autoestima, onde pode ser representado como sentimento, o apreço e a consideração que uma pessoa sente por si própria, se encontra associada aos aspectos avaliativos que o sujeito elabora a seu respeito, baseando nas suas capacidades e desempenho.

Deste modo, infere-se que a autoestima e consequentemente a imagem corporal, pode apresentar relação com a sexualidade dos idosos. Destaca-se que a literatura tem evidenciado que a postura destes ainda é conservadora acerca desta temática. Este fato pode estar relacionado a educação no passado contribuindo para gerar dúvidas, medos e preconceitos dificultando a vivência da sexualidade nesta etapa da vida ${ }^{(12)}$.

Alguns dos motivos que contribuem para o aumento e reprodução de uma visão errônea sobre a sexualidade na velhice são a falta de conhecimento acerca do tema na velhice, embora isto não se restrinja apenas a essa fase da vida, assim como ideologias que não incentivam a expressão da sexualidade entre as idosas ${ }^{(13)}$. Outro fator que impede uma relação amistosa com a sexualidade na velhice é a educação repressora e a associação que a sociedade faz entre atividade sexual e procriação ${ }^{(14)}$.

Nesta revisão de literatura, idosas que tinham uma vida mais discreta, consequentemente não demonstraram sua necessidade por uma vida sexual ativa. Elas também se preocupavam com sua imagem corporal, para a manutenção de uma postura discreta tão desejada e normatizada pela vida social e consequentemente pela sociedade. Nos seus relatos as idosas sentem-se esmagadas pelos preconceitos e alimenta o pensamento de que por ser "velha" já não oferece atrativos. Nessa perspectiva, opta por uma postura mais discreta para não ser rotu- lada como a "velha assanhada". Isso se aplica, obrigatoriamente, às vestimentas e ao modo de se comunicar ${ }^{(15)}$.

A imagem corporal também é relatada pela insatisfação relacionada ao aumento do peso, principalmente nas mulheres, conduzindo que as mesmas sejam mais críticas com sua aparência física, levando à diminuição ou à ausência nas relações afetivas com o outro, condicionadas pela não aceitação de si e, com isso, a alteração do desejo sexual e consequente recusa para 0 sexo ${ }^{(16)}$.

A vergonha do corpo refere-se aos sentimentos negativos que ocorrem quando as pessoas se avaliam contra os ideais culturais internalizados de aparência e descobrem que ficam aquém desses ideais'. A consequência é a negação da sexualidade dessas idosas, na qual a sociedade é forte contribuinte ao atribuí-la apenas como direito dos jovens e adultos. A sexualidade das idosas apenas será vivida de forma completa e livre quando novas percepções sociais forem concretizadas, garantindo a vivência erótica em qualquer fase da vida ${ }^{(15)}$.

A outra visão mostrada pelo segundo artigo seria de que a imagem corporal não influenciava no desejo e na satisfação sexual, mesmo com os corpos envelhecidos, elas ainda podiam ser desejáveis. Corroborando com essa afirmação, algumas idosas citam que: "“pra mim, meu corpo não fica velho. Eu sinto até o amor [desejo sexual]." (sra. P, 70 anos); estou feliz com meu corpo, a transformação é natural; só fiquei assim ressecada". (sra. C, 70 anos). ${ }^{(19)}$

Percebe-se que a ligação da imagem corporal com a sua sexualidade é algo intimo de cada pessoa e que está atrelado aos fatores de ordens biológicas, psicológicas e socioculturais ${ }^{(20)}$. Outras razões que podem levar ao comprometimento da satisfação sexual seria a qualidade de vida e o próprio relacionamento (por exemplo, comunicação, intimidade emocional, respeito, companheirismo, satisfação conjungal), onde demonstraram serem, importantes preditores, inclusive mais do que a imagem corporal, para as mulheres citadas no estudo(21).

Nesse contexto, para as idosas que relatam influencia da imagem corporal com a sexualidade, é necessário que haja um processo de educação sexual direcionado para as pessoas que estão na terceira idade, a fim de reeducá-las, proporcionando-lhes bem-estar, qualidade de vida, auto-conhecimento, trabalho com as questões acerca da imagem corporal, muitas vezes mutilada nesse momento da vida em nossa sociedade, bem como em relação às novas possibilidades e elaboração de projetos de vida. Para além de um ganho pessoal, esses idosos podem tornar-se grandes exemplos para os mais jovens com os quais convivem ${ }^{(17)}$.

\section{CONCLUSÃO}

Os mitos sobre o envelhecimento e a sexualidade se constituem um dos desafios que os idosos enfrentam e 
que os proíbe de viver intensamente sua sexualidade. $O$ fato é que a maior parte da sociedade continua concebendo a sexualidade como uma capacidade submetida ao desgaste dos anos, sendo prerrogativa dos mais jovens e isso provoca nas pessoas de mais idade uma perda de interesse por ela.

Além do preconceito diante da sexualidade nas pesso- as idosas, a sociedade impõe um padrão de corpo perfeito para as mulheres e obviamente, isso compromete a sua auto-estima e a sua percepção da imagem corporal, e consequentemente o rendimento na qualidade da vida sexual.

Porém, percebe-se que mesmo com a imposição do corpo perfeito, em alguns casos, não existe a influencia da sexualidade em idosas. 


\section{REFERENCIAS}

I. Shkolnik D, Lecovich E. Health, body image, gender, and migration status: their relationship to sexuality in old age. International Psychogeriatrics [Internet] 2013 [acesso em 20 de abril 2020]; 25 (I0), |7|7-I727.Disponível em:https://www.cambridge.org/ core/journals/international-psychogeriatrics/article/health-body -image-gender-and-migration-status-their-relationship-to-sexuality-in-old-age/A74F99B7C2D I735620AA27593 IEBDE28

2. Lves D, Pinto M, Alves S, Mota A, Leirós V. Cultura e imagem corporal. Motri. [Internet] 2009 [acesso em 20 de abril 2020]; 5(I): I-20. Disponível em: http://www.revistamotricidade.com/arquivo/2009_vol5_nl/v5n la02.pdf

3. Fernandes MGM. Problematizando o corpo e a sexualidade de mulheres idosas: o olhar de gênero e geração. Rev. enferm. [Internet] 2009 [acesso em 20 de abril 2020]; I7 (3): 418-22. Disponível em: https://pesquisa.bvsalud.org/portal/ resource/pt/bde-17059

4. Bourdieu P.A dominação masculina. Rio de Janeiro: Bertrand Brasil,2005.Disponível em:https://edisciplinas.usp.br/pluginfile. php/3026074/mod_resource/content/I/BOURDIEU\%2C\%20 P.\%20A\%20Domina\%C3\%A7\%C3\%A30\%20Masculina.pdf

5. Browna DL, Bradleyb TW, Cobbc PD, Spawa D,Aldridgea KN. African American women's safer sexual practices: the influence of ethnic-racial socialisation and body esteem. Culture, Health \& Sexuality [Internet] 2014 [acesso em 20 de abril 2020]; I6(5): 5 I 8-532. Disponível em: https://pubmed. ncbi.nlm.nih.gov/24654840/

6. Robbins AR, Reissing ED. Appearance Dissatisfaction, Body Appreciation, and Sexual Health in Women Across Adulthood.Arch Sex Behav [Internet] (2017) [acesso em 20 de abril 2020]; 6(2): I 3-28. Disponível em: https://pubmed.ncbi. nlm.nih.gov/28378092/

7. Beyea SC, Nicoll LH.Writing an integrative review.Aorn ] [Internet] 1998 [acesso em 20 de abril 2020]; Apr; 67(4):87780. Disponível em: https://pubmed.ncbi.nlm.nih.gov/9616108/

8. Keynes M. Primary Care Trust. Critical Appraisal Skills Programme. London: Oxford; 2002. Disponível em: https://casp-uk.net/

9. Stillwell S, Melnyk BM,Williamson K. Evidence-based practice: step by step. Am J Nurs [Internet] 2010 [acesso em 20 de abril 2020]; I 10(5):4 I-7. Disponível em: https://pubmed. ncbi.nlm.nih.gov/21 709484/

I0. Thorpea R, Fileborna B, Hawkesb G, Pittsa M, Minichiello V. Old and desirable: older women's accounts of ageing bodies in intimate relationships. Journal Sexual and Relationship Therapy [Internet] 2015, [acesso em 02 de junho 2020]; 30 (I). Disponível em: https://www.tandfonline.com/ doi/abs/I0.1080/14681994.2014.959307

II. Vieira KFL, Coutinho MPL, Saraiva ERA. A Sexualidade Na Velhice: Representações Sociais De Idosos Frequentadores de Um Grupo de Convivência. Psicol. Cienc [Internet] 2010, [acesso em 20 de abril 2020]; 36 (I)I96209. Disponível em: https://www.scielo.br/scielo.php?pi$\mathrm{d}=S|4| 4-989320|6000| 00 \mid 96 \&$ script=sci_abstract\&tlng=pt
12. Ranuzi C, Santos TG, Rodrigues LR, Fonseca LRM, Malaquias BSS. Sexualidade e autoestima em idosos. Anais V CIEHV. I, 20 I7. Disponível em: https://editorarealize.com.br/revistas/ cieh/anais.php

13. Frugoli A, Magalhães CAO.A sexualidade na terceira idade na percepção de um grupo de idosas e indicações para a educação sexual. Arq. Ciênc. Saúde UNIPAR, Umuarama [Internet] 20 I I [acesso em 20 de abril 2020]; I 5( I):85-93. Disponível em: https://revistas.unipar.br/index.php/saude/article/view/3696

14. Risman A. Sexualidade e terceira idade: uma visão históricocultural. In: Textos sobre envelhecimento [Internet] 2005 [acesso em 20 de abril 2020]; 8 (I), I5-2I. Disponível em: http://revista.unati.uerj.br/scielo.php?script=sci_arttext\&pid=S I I | 7-59282005000 I 00006\&lng=pt\&nrm=iso

15. Souza M, Marcon SS, Bueno SMV, Carreira L, Baldissera VDA. A vivência da sexualidade por idosas viúvas e suas percepções quanto à opinião dos familiares a respeito. Saúde Soc [Internet] 2015 [acesso em 20 de abril 2020]; 24 (3): 936-944. Disponível em: https://www.scielo.br/pdf/sausoc/ v24n3/0 I04-I290-sausoc-24-03-00936.pdf

16. Oliveira LB, Baía RV, Delgado ART,Vieira KFL, Lucena ALR. Sexualidade e Envelhecimento: Avaliação do Perfil Sexual De Idosos Não Institucionalizados. Rev. Ciênc. Saúde Nova Esperança. [internet] 2015 [acesso em 20 de abril 2020]; 13 (2):42-50. Disponível http://www.facene.com.br/wpcontent/uploads/20 I0/I I/SEXUALIDADE-E-ENVELHECIMENTO-PRONTO.pdf.

17. Siqueira TCB, Pereira ABM. Fragmentos de cultura [Internet] 2007 [acesso em 20 de abril 2020]; I7(3): 27I-277.

18. Polit DF, Beck CT, Hungler BP. Fundamentos de pesquisa em enfermagem: métodos, avaliação e utilização. $6^{\mathrm{a}}$ ed. Porto Alegre:Artmed; 2006.

19. Fernandes MGM, Garcia LG. O corpo envelhecido: percepção e vivência de mulheres idosas. Interface - Comunic. Saúde Educ [Internet] 2010 [acesso em 20 de abril 2020]; I4(35): 879-90. Disponível: https://www.scielo.br/scielo.php?pi$d=S|4| 4-328320100004000 \mid$ 3\&script=sci_abstract\&tlng=pt.

20. Oliveira DM, Jesus MCP,Merighi MAB. Climatério e sexualidade:A compreensão dessa interface por mulheres assistidas em grupo. Texto Contexto Enfermagem [Internet] 2008 [acesso em 20 de abril 2020]; I7(3): 5 I 9-26. Disponível: https://www. scielo.br/scielo.php?pid=s0 I 04070720080003000 I 3\&scrip$\mathrm{t}=$ sci_abstract\&tlng=pt.

2I. Koch PB, Mansfield PK, Thurau D, Carey M."Feeling frumpy”:The relationships between body image and sexual response changes in midlife women. The Journal of Sex Research [Internet] 2005 [acesso em 20 de abril 2020]; 42(3): 215-223. Disponível em: https://www.researchgate.net/publication/26882904_Feeling Frumpy_The_Relationships_between_Body_Image_and_Sexual_Response_Changes_in_Midlife_Women

Recebido: 2019-1 I-14

Aceito: 2020-04-20 